\title{
Development and Implementation of a Synchronous Online TBL Using Microsoft Forms
}

\author{
Courtney E. Cross ${ }^{1}$ (D) $\cdot$ Christina Robinson ${ }^{1,2} \cdot$ Emily Todd $^{1}$
}

Accepted: 19 October 2020 / Published online: 24 October 2020

(C) International Association of Medical Science Educators 2020

\begin{abstract}
After transitioning to a virtual flipped classroom due to COVID-19, pre-clinical content application session facilitators noted decreased engagement in large group sessions. Thus, we developed a synchronous virtual team-based learning session using Microsoft Forms. Students identified increased engagement, deeper learning, and ease of technology use as benefits to online team-based learning.
\end{abstract}

Keywords Team-based learning $\cdot$ Flipped classroom $\cdot$ Virtual classroom $\cdot$ Student engagement $\cdot$ Educational technology

Virtual learning presents challenges to many aspects of medical education. However, COVID-19 necessitated our school transition to a virtual learning environment in March 2020. Our pre-clinical curriculum is flipped classroom, which we continued using Zoom. Facilitators noted decreased engagement within the online platform, so our team developed a method to simulate team-based learning (TBL) within the virtual learning environment utilizing breakout rooms. Using the TBL collaborative (TBL-C) white paper outlining best practices for online TBL, we identified the following areas of focus: promoting learning, timely feedback, and maintaining the TBL structure of orientation, readiness assurance, and application exercises [1].

Pre-classroom Development In order to mimic the IF-AT cards and the $4 \mathrm{~S}$ application exercises of TBL, we used Microsoft Forms to develop the iRAT, tRAT, and application questions, and Excel for automatic reporting. The iRAT was a multiple-choice quiz, without the correct answers reported to the student. The tRAT was developed using point allocation and branching. Each question was inputted to Forms 3 times,

Courtney E. Cross

courtneycross@tcu.edu

1 Department of Medical Education, TCU \& UNTHSC School of Medicine, Fort Worth, TX, USA

2 Department of Pediatrics, University of Texas Health Science Center, Fort Worth, TX, USA and given 4, 2, or 1 points. A correct answer triggered an automated response congratulating the group for a correct answer and assigning the appropriate number of points. An incorrect answer allowed students to try again. After three attempts, the group was given the correct answer and advanced to the next question. The point allocation served as a motivator to students and allowed facilitators to track attempts required to answer the question correctly by each group. An Excel spreadsheet was used to generate a matrix flagging challenging questions and graphing the average points per question to facilitate discussion in the full group (Fig. 1). Likewise, 4S application exercises were built in Forms using the basic quiz format, without the correct answer reported to the students. An Excel workbook was built to convert the answer data into pie charts imbedded in PowerPoint (Fig. 1). The links for all activities were posted on Canvas and released to the students just prior to class.

Orientation Our school utilized TBL before transitioning to virtual learning, so orientation focused on communication and technology. The classroom flow began with students and facilitators on Zoom as a large group with the agenda, content framing, and instructions for the session provided by a shared screen.

Readiness Assurance Process Students first completed the iRAT. Students then transitioned to breakout rooms and completed the tRAT. Facilitators were pushed into breakout rooms to provide support. Within each breakout room, one student opened the tRAT, shared their screen, and submitted answers 
a tRAT Question Point Allocation Matrix for Faculty Use

\begin{tabular}{|c|c|c|c|c|c|c|c|c|c|c|c|c|c|c|c|c|c|}
\hline & \multicolumn{17}{|c|}{ Question Number } \\
\hline & 1 & 2 & 3 & 4 & 5 & 6 & 7 & 8 & 9 & 10 & 11 & 12 & 13 & 14 & 15 & 16 & 17 \\
\hline Group 1 & 4 & 4 & 4 & 4 & 4 & 4 & 4 & 4 & 4 & 4 & 4 & 4 & 4 & 0 & 4 & 2 & 4 \\
\hline Group 2 & 4 & 4 & 4 & 4 & 4 & 4 & 4 & 4 & 4 & 4 & 4 & 4 & 4 & 4 & 4 & 4 & 4 \\
\hline Group 3 & 4 & 1 & 4 & 4 & 4 & 4 & 4 & 4 & 4 & 4 & 4 & 4 & 4 & 1 & 4 & 4 & 2 \\
\hline Group 4 & 4 & 1 & 4 & 4 & 4 & 4 & 4 & 4 & 4 & 4 & 4 & 4 & 4 & 1 & 4 & 4 & 4 \\
\hline Group 5 & 4 & 4 & 4 & 4 & 4 & 4 & 4 & 2 & 4 & 4 & 4 & 4 & 4 & 0 & 4 & 4 & 4 \\
\hline Group 6 & 4 & 4 & 4 & 4 & 4 & 4 & 4 & 4 & 4 & 4 & 4 & 4 & 4 & 2 & 4 & 4 & 1 \\
\hline Group 7 & 4 & 4 & 4 & 4 & 4 & 4 & 4 & 4 & 4 & 4 & 4 & 4 & 4 & 4 & 4 & 4 & 4 \\
\hline Group 8 & 4 & 1 & 4 & 4 & 4 & 4 & 4 & 4 & 4 & 4 & 4 & 4 & 4 & 1 & 4 & 4 & 4 \\
\hline Group 9 & 4 & 4 & 4 & 4 & 4 & 4 & 4 & 4 & 4 & 4 & 4 & 4 & 4 & 1 & 4 & 4 & 2 \\
\hline Group 10 & 4 & 1 & 4 & 4 & 4 & 4 & 4 & 4 & 4 & 4 & 4 & 4 & 4 & 1 & 4 & 4 & 2 \\
\hline
\end{tabular}

b tRAT Score Graph for Classroom Use

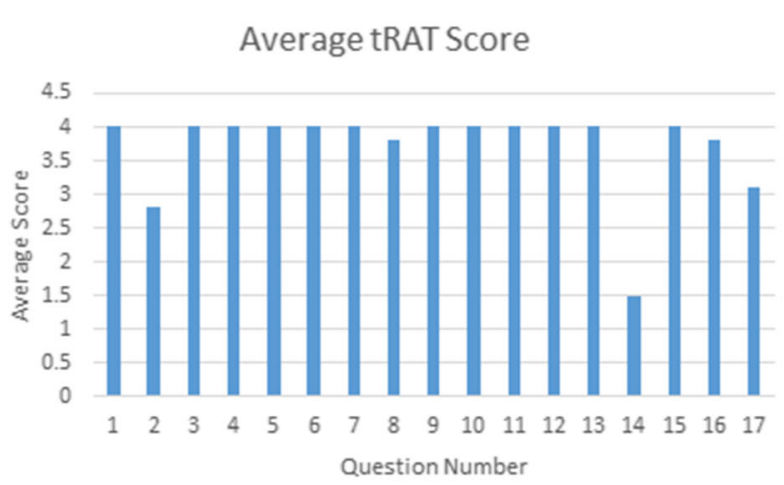

Fig. 1 Excel output for the tRAT Point Allocation Matrix, Class Scores, and Application Response Chart. The matrix is for faculty use only and collates data from the Microsoft Forms output to score each question in the same method as IF-AT cards -4 points for a correct answer on the

on behalf of the group. During the transition back to the large group, the tRAT data was downloaded from Forms and applied to the Excel workbook. Facilitators then discussed challenging RAT questions and answered student-generated questions that peers could not answer during the tRAT.

Application Exercises Students were sent back to their breakout rooms to complete application exercises, and then returned to the large group for discussion at which time the Forms data was imported to Excel. A pie chart of the group responses was provided by shared screen as the application exercises were deliberated in the large group. Students of opposing incorrect and correct answers were invited to share the reasoning for their group's response. Facilitators then provided further discussion points. In this way, we met the significant problem, same problem, and specific choice guidelines for application exercises. Although we could not have simultaneous reporting, the pie charts with student responses guided discussion.

Reflection and Evaluation Facilitators reported more student engagement during this online TBL session than in other virtual instruction sessions. The student evaluation responses are
C Application Question Pie Chart Responses for Classroom Use

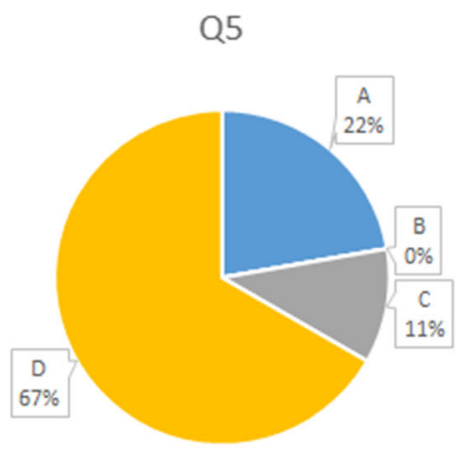

first attempt, two for the second, etc. The Class Scores and Application Response Chart allows students to see the score by question and facilitates discussion

not able to be included in this document but were overall favorable. The main themes identified by students were increased engagement and preparedness, appropriately challenging questions, a deeper level of learning in the breakout rooms, and ease of use with the technology. Students identified that a hard copy of the iRAT questions would have been helpful to use during the tRAT.

Further Development Since we anticipate remaining in the virtual classroom environment for the pre-clinical curriculum through at least January 2021, we have modified our virtual TBL prototype and written a user guide for all faculty to utilize. The tRAT Forms document was converted into a template, with 20 questions $/ 5$ choices per question. All branching sites were pre-programmed and points pre-assigned. The tRAT calculations Excel spreadsheet was also converted into a template, so that faculty only need to insert the tRAT Microsoft Forms data to generate the matrix data and graph.

Authors' Contributions 1)Made substantial contributions to the conception or design of the work; or the acquisition, analysis, or interpretation of data; or the creation of new software used in the work- $\mathrm{CC}, \mathrm{CR}$, and ET contributed equally to design of the session and question writing. $\mathrm{CC}$ developed the Excel spreadsheet templates. 
2)Drafted the work or revised it critically for important intellectual content - $\mathrm{CC}$ and $\mathrm{CR}$ contributed equally to the initial draft. ET was responsible for editing and revisions.

3)Approved the version to be published-CC, CR, and ET all reviewed and approved the final version.

4)Agree to be accountable for all aspects of the work in ensuring that questions related to the accuracy or integrity of any part of the work are appropriately investigated and resolved-CC, CR, and ET all agree to be accountable for all aspects of the work.

Data Availability Not applicable.

\section{Compliance with Ethical Standards}

Conflict of Interest Courtney Cross is a faculty reviewer for USMLERx, which includes First Aid for the USMLE and ScholarRx.

Ethics Approval Not applicable.

Consent to Participate Not applicable.
Consent for Publication Not applicable.

Code Availability Not applicable.

\section{Reference}

1. Clark M, Merrick LC, Styron JL, Dolowitz AR, Dorius C, Madeka K, Bender H, Johnson J, Chapman J, Gillette MT, Dorneich MC, O'Dwyer B, Grogan J, Brown TJ, Leonard B, Rongerude JM, Winter L. Off to on: best practices for online team-based learning. In: TBL Online, Team-Based Learning ${ }^{\mathrm{TM}}$ Collaborative. 2018. http://www.teambasedlearning.org/wp-content/uploads/2018/08/ Off-to-On_OnlineTBL_WhitePaper_ClarkEtal2018_V3.pdf. Last accessed 28 June 2020.

Publisher's Note Springer Nature remains neutral with regard to jurisdictional claims in published maps and institutional affiliations. 